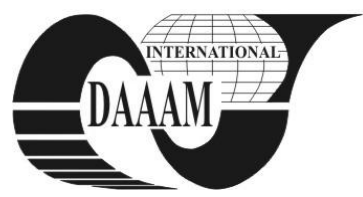

Annals of DAAAM for 2011 \& Proceedings of the 22nd International DAAAM Symposium, Volume 22, No. 1, ISSN 1726-9679 ISBN 978-3-901509-83-4, Editor B. Katalinic, Published by DAAAM International, Vienna, Austria, EU, 2011 Make Harmony between Technology and Nature, and Your Mind will Fly Free as a Bird Annals \& Proceedings of DAAAM International 2011

\title{
A COMPARATIVE STUDY OF A REAL SYSTEM IDENTIFICATION
}

\author{
PISAN, R[adim] \& GAZDOS, F[rantisek]
}

\begin{abstract}
This article compares two methods of identification of a real system. The first one is the classical Least squares method and the second one is a Neural network approach that uses the feedforward network with tree layers. The LevenbergMarquardt was chosen as the learning algorithm. Laboratory equipment Amira DR300 was selected for the verification of both methods. It is a servo-mechanism which consists of a permanently excited DC-motor and a tacho-generator with the incremental encoder as a sensor of the output signal
\end{abstract}

Key words: identification, neural network, least squares method, AMIRA DR 300

\section{INTRODUCTION}

Identification is a process where the measured input and output data of an unknown system is at our disposal and we want to describe behavior of this system mathematically. It is possible to use the classical methods of identification, e.g. the least squares method (Ljung, 1999) or we can use a neural network as artificial intelligence (Dreyfus, 2005). The network is composed of an input layer, hidden layers and an output layer. The input terminal is represented by the input data set. A simple neuron is composed of a transfer function, for instance, the linear function, the log-sigmoid, the tan-sigmoid and of the sum of multiples of corresponding inputs and their weights. The network has two cycles. The first one is called forward where the input data are recalculated over all layers and the output is also calculated. This value is compared with the measured output of the identified system and the error is determined (Norgaard et al., 2000). Then the value is propagated back over the whole network and the weights are updated. This process is called learning of the network. Several learning algorithms were developed during last decades, e.g. the Backpropagation, the Levenberg-Marquardt (Hagan \& Menhaj, 1994), etc. In this paper, experimental identification of a real system was performed by two different approaches - using the Least Squares Method (LSM) and by means of a Neural Network (NN). Achieved results is further compared and discussed.

\section{AMIRA SYSTEM DR300}

The identified system is represented by a permanently exited DC-motor of which the input signal (armature current) is provided by a current control loop. Its servo amplifier allows the 4-quadrant mode. Sensors for the output signal (revolutions) are represented by a tacho-generator and an incremental encoder. The free end of the motor shaft is coupled fixedly to the shaft of a second, identical motor. This motor is used as a generator. Its output current is freely adjustable $(* * *)$. The range of the input signal is from -0.25 to 0.25 Matlab Unit (MU) and the output is measured from -1 to 1 . The device has also area of lower and upper saturation. Static characteristic of the system is presented in the Fig. 1. where one quadrant was measured only once, i.e., only positive revolutions were

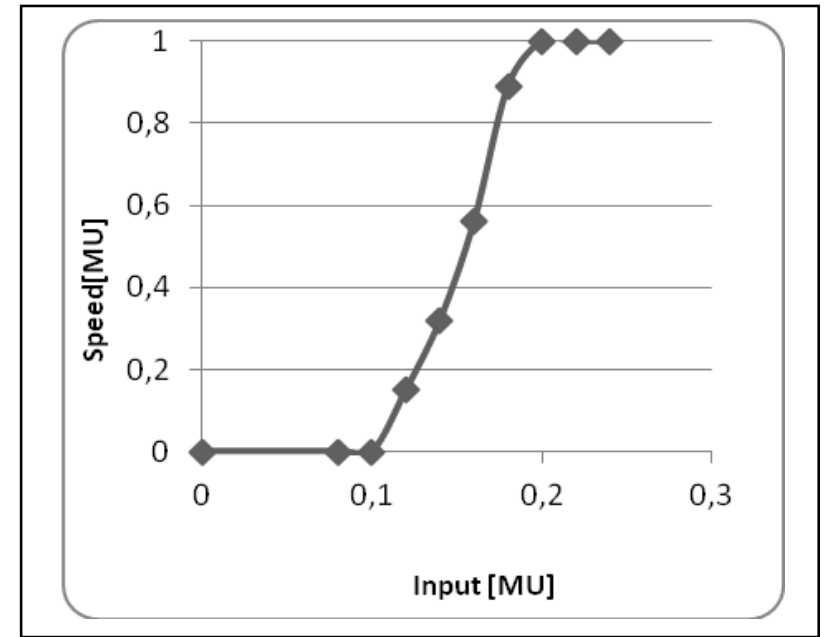

Fig. 1. The static characteristic of the Amira system DR 300

measured. Step responses were applied to the system and the output signal was obtained in the steady state.

\section{APPLIED NEURAL NETWORK}

The feedforward network presented in Fig. 2 was chosen for the experiment. This network has one hidden layer, one input layer and one output layer. The input layer has four terminals, the hidden layer is constituted of 4 neurons and output layer forms one neuron. The transfer function in the hidden layer is the logsigmoid and a linear function is in the output. The random signal was used for the training data set. This signal was given to the input of the Amira system and the response was obtained. Further, the off-line learning method with the MarquardtLevenberg algorithm was used. This algorithm is based on the Gauss-Newton method. Fig. 3 shows structure of the training process. Here, the blocks $\mathrm{z}^{-1}$ and $\mathrm{z}^{-2}$ denote one and two-step delays respectively. The learning parameters were following:

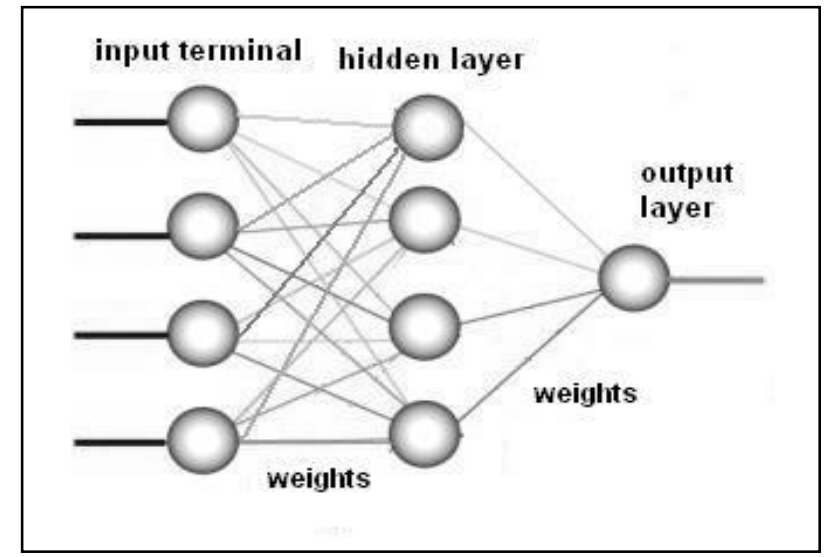

Fig. 2. Structure of the Neural network 


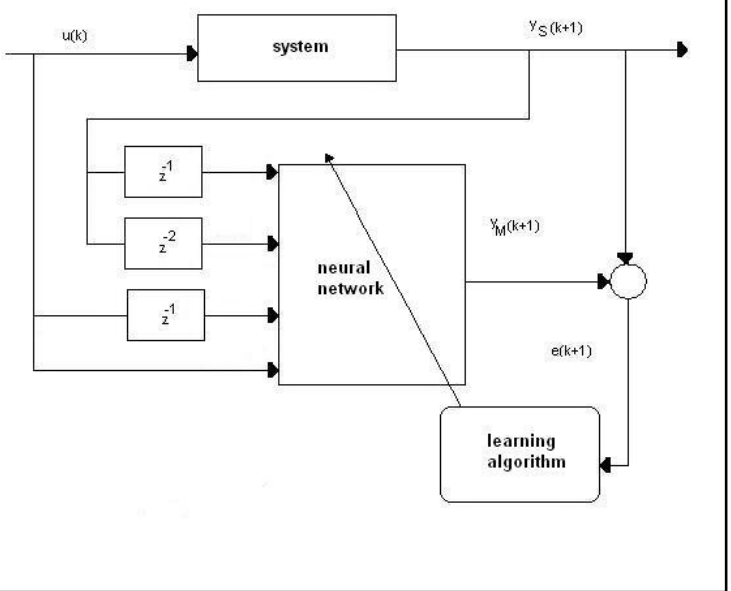

Fig. 3. Learning Neural network

2500 epochs, minimal global error: $1 \mathrm{e}-7$ and a number of data set: 48685 .

\section{THE LEAST SQUARES METHOD}

The classical least squares method was chosen as the second algorithm for the system identification. First, structure of the model was chosen as a discrete transfer function:

$$
G(z)=\frac{b_{1} z+b_{0}}{z^{2}+a_{1} z+a_{0}}
$$

Hence, it is a $2^{\text {nd }}$ order system with unknown parameters $b_{1}$, $b_{0}, a_{1}, a_{0}$. These parameters were further calculated as:

$$
\widehat{\Theta}=\left[\Psi^{T}(k) \Psi(k)\right]^{-1} \Psi^{T}(k) y(k)
$$

where $\Psi(k)$ is a data matrix, $y(k)$ is a vector of output and $\widehat{\Theta}$ is a vector of these unknown parameters estimates.

For determination of the parameters estimates we have to select a working point and then measure input/output data around this point and use the formula (2).

\section{COMPARISON OF THE RESULTS}

A working point located in the center of the static characteristic was chosen as the system behaviour is linear in this area. The random input signal was applied near this point. Because the system has static characteristic with saturation, we had to adjust the data for the LSM algorithm. The value of lower saturation was subtracted from the input data and the resultant transfer function (1) was determined using (2) as:

$$
G(z)=\frac{0.5717 z+0.2191}{z^{2}-0.7093 z-0.2123}
$$

For comparison we have to measure test data. Two step responses of the obtained model (3) were recorded for this purpose. The same input data were applied to the input terminal of the neural network and the response was calculated. Further, a real response of the Amira system was also measured. Obtained data were compared using this formula:

$$
E=\frac{1}{N} \sum_{n=1}^{N}\left(o_{r_{n}}-o_{a_{n}}\right)^{2}
$$

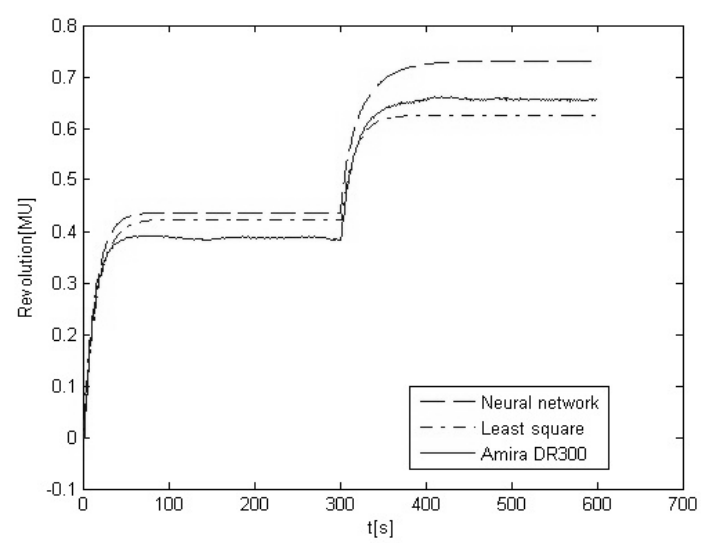

Fig. 4. Comparison of the identification results

where $\mathrm{N}$ is a total number of samples, $\mathrm{o}_{\mathrm{r}}$ is $\mathrm{n}$-th real output and $\mathrm{o}_{\mathrm{a}}$ is $\mathrm{n}$-th Neural network or Least squares output.

The figure above (Fig. 4) shows step responses of the Neural network model, Least squares model and of the real Amira system. The resultant deviation between the NN model and Amira system was evaluated as $3.4 \times 10^{-3}$ whereas the deviation between the LSM and Amira was calculated as $9.2 * 10^{-4}$. The results, therefore, demonstrate that the LSM approximate is in this case better then the obtained NN model.

\section{CONCLUSION}

The aim of this article was to compare two identification approaches for the real device Amira DR300. The Neural network and the Least squares method were the competitors. Firstly, the static characteristic was measured. This has upper and lower saturation which is a problem for the LSM as the input data has to be adjusted. The neural network does not face this problem and it can approximate the system in the whole range. In this case a small, linear part of the static characteristic was chosen and step responses were measured for the comparison. The results show, overall, that the LSM has smaller deviation from the real system behaviour then the NN. However, the advantage of Neural networks is in its capability to describe a considerable non-linearities, e.g. saturation. The main disadvantage is in determination of a suitable training set and its number together with an appropriate sample time.

\section{ACKNOWLEDGEMENTS}

This work was supported by the Internal Grant Agency of Tomas Bata University in Zlin, project no. IGA/55/FAI/10/D.

\section{REFERENCES}

Dreyfus, G. (2005) Neural networks methodology and applications, Editions Eyerolles, ISBN: 978-3-540-22980-3 Hardcover

Hagan, M.; Menhaj, M.(1994), Training Feedforward Networks with the Marquardt Algorithm. Transactions of neural network, 5, 6, 989-993

Ljung, L. (1999) System Identification: Theory for the User, Prentice Hall, Englewood Cliffs, New Jersey

Norgaard, M., Ravn, O., Poulsen, N.K. \& Hansen, L.K. (2000), Neural networks for modelling and control of dynamic systems, Softcover, ISBN: 978-1-85233-227-3

*** (2006) http://www.amira.de/dr300_engl.html -Amira measuring and control technology, Laboratory equipment Accessed on: 2011-06-13 